\title{
Experimental determination of the primary trigeminal projections in lampreys
}

\section{R. GLENN NORTHCUTT}

Division of Biological Sciences, The University of Michigan, Ann Arbor, Mich. 48109 (U.S.A.)

(Accepted November 9th, 1978)

The trigeminal complex of lampreys, like that of anamniotic gnathostomes, consists of profundus and 'mandibulo 'mandibulomaxillary' branches ${ }^{\mathbf{3 , 6}}$. The profundus branch possesses a distinct ganglion (supraorbital ganglion) separate from the ganglion (suboptical ganglion) of the 'mandibulomaxillary' branches ${ }^{12}$. However, both ganglia send their processes into the medulla as a single trigeminal sensory root (Figs. 1A, 2A). The lamprey trigeminal complex differs from that of other anamniotes in that the anterior lateral line and facial ganglia are not closely associated with the trigeminal ganglia but are located beneath and within the optic capsule ${ }^{3,12}$. This separation clearly simplifies experimental analysis of the trigeminal projections, as the trigeminal ganglia can be lesioned or the sensory roots transected without damaging facial or lateralis inputs.

Earlier studies claim that scattered lateral line receptors on the dorsal surface of the head are innervated by the trigeminal nerve ${ }^{2,5}$. This condition has not been reported for any other vertebrate and has been interpreted as supporting the hypothesis that a lateralis component existed ancestrally in each branchiomeric cranial nerve. It is also claimed that the trigeminal nerve in lampreys projects directly to the cerebellum, the octavolateralis area and the funicular nucleus (presumed precursor of the dorsal column nuclei ${ }^{4,6}$ ). This trigeminal projection pattern has been interpreted as supporting the claim that the dorsal column nuclei, octavolaterlis area and cerebellum arose from a single somatic sensory column, and that lampreys essentially retain that primitive condition ${ }^{6}$. Finally, a distinct mesencephalic trigeminal nucleus has not been identified in lampreys and is assumed to have arisen with the evolution of jawed vertebrates. These claims are of considerable importance regarding the evolution of the trigeminal system and were the impetus for the present study.

The trigeminal ganglia in 5 transformed specimens (11-16 cm in length) of Ichthyomyzon unicuspis were unilaterally destroyed by transection or heat cauterization of the ganglia under MS222 anesthesia. The animals survived 3, 5, 7 and 12 days at $23{ }^{\circ} \mathrm{C}$ before they were reanesthetized, the brains dissected from the neurocranium and emersed in $10 \%$ formalin. The brains were embedded in $25 \%$ gelatin and processed by the Wiitanen procedure ${ }^{14}$ for demonstrating degenerating axons and their terminals. All the survival times were adequate to reveal degenerating trigeminal 


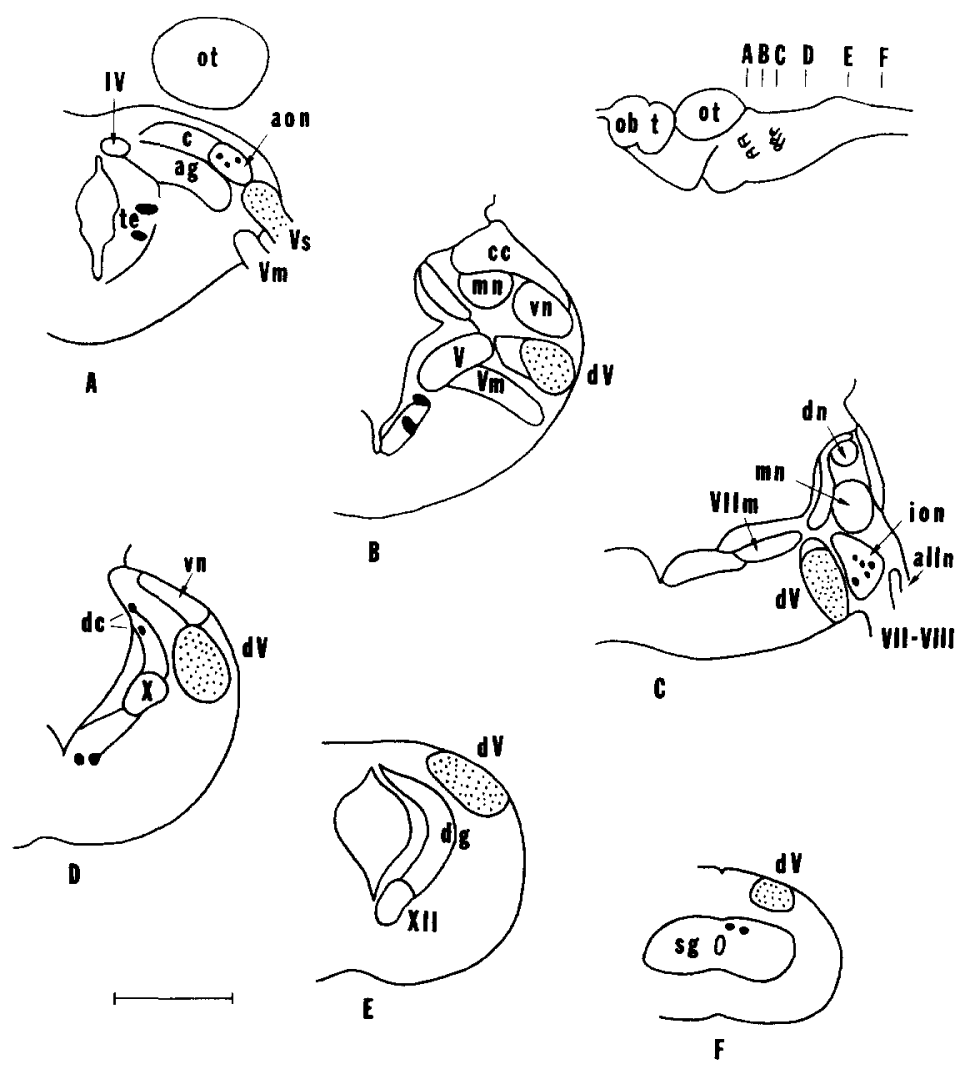

Fig. 1. Charting of the degenerating trigeminal afferents following transection of the trigeminal sensory root. A-F: transverse sections through the hindbrain at levels indicated in the lateral view of the brain. Fine stippling indicates degenerating fibers and terminals, large solid black circles indicate individual large neurons. Bar scale equals $500 \mu \mathrm{m}$. Abbreviations: ag, alar gray; alln, anterior lateral line nerve; aon, anterior octavomotor nucleus; c, cerebellum; cc, cerebellar crest; dc, dorsal cells; dg, dorsal gray; $\mathrm{dn}$, dorsal octavolateralis nucleus; $\mathrm{dV}$, descending trigeminal tract and nucleus: ion, intermediate octavomotor nucleus; mn, medial octavolateralis nucleus; ob, olfactory bulb; ot, optic tectum; sg, spinal gray; t, telencephalon; te, tegmentum; vn, ventral octavolateralis nucleus; IV, trochlear motor nucleus; $\mathrm{V}$, trigeminal motor nucleus; $\mathrm{Vm}$, trigeminal motor root; $\mathrm{Vs}$, trigeminal sensory root; $\mathrm{VIIm}$, facial motor nucleus; VII, facial root; VIII, octavius root; X, vagal motor nucleus; XII, hypoglossal motor nucleus.

fibers, with 5 and 7 days being optimal. The distribution of the degenerating trigeminal fibers is charted in Fig. 1. The nomenclature is that of Nieuwenhuys ${ }^{10,11}$ except for the term medial octavolateralis nucleus rather than his intermediate octavolateralis nucleus.

The degenerating trigeminal sensory fibers enter the hindbrain, at the level of the isthmus (Figs. 1A, 2A, C), as the dorsal sensory root of the trigeminal nerve. The fibers turn caudally, forming the descending trigeminal tract (Figs. 1B, 2B) which is located ventrolaterally beneath the octavolateralis area throughout most of the extent of the hindbrain (Fig. 1B-D). Degenerating trigeminal fibers were seen only ipsilateral to the lesioned ganglia. Neurons scattered among the fibers of the descending tract along its 

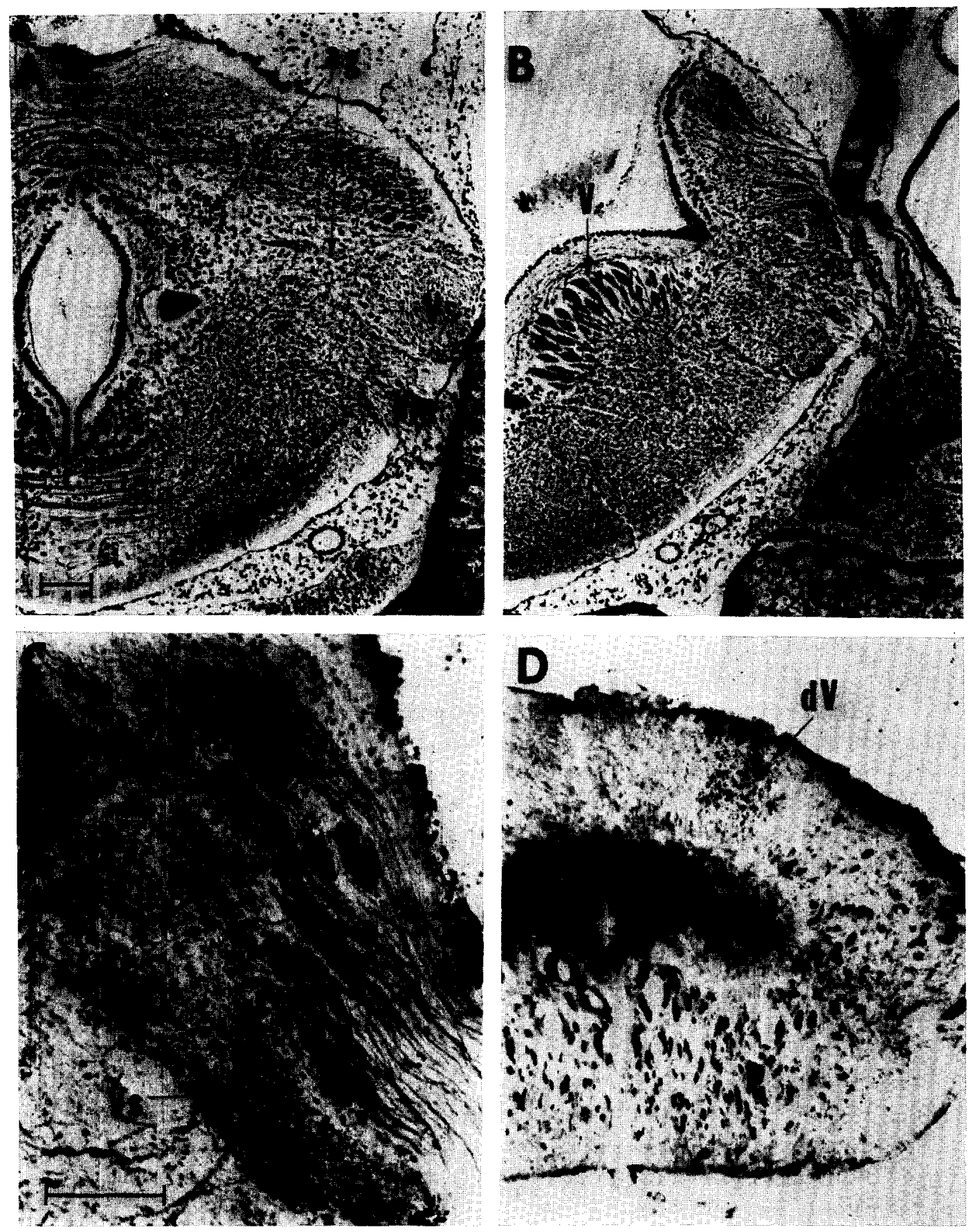

Fig. 2. Photomicrographs of lamprey hindbrain and spinal cord. A: transverse section through the cerebellum and isthmus at the level of the trigeminal sensory and motor roots. B: transverse section through the medulla at the level of the trigeminal motor nucleus and descending trigeminal tract (outlined by dashed line). $C$ : transverse section at an isthmal level showing the degenerating trigeminal sensory root. D: transverse section of the spinal cord showing the degenerating descending trigeminal tract in the dorsal funiculus. Bar scales equal $100 \mu \mathrm{m}$. Magnifications of A and B are identical, as are magnifications of $C$ and $D$. Abbreviations: ag, alar gray; $d V$, descending trigeminal tract; $V$, trigeminal motor nucleus; Vs, trigeminal sensory root; $\mathrm{Vm}$, trigeminal motor root. 
entire length, and along its medial edge, constitute a descending trigeminal nucleus (Fig. 2A, B).

As the obex is approached, the descending trigeminal tract moves dorsally (Fig. 1E) to occupy the medullar region previously described as the funicular nucleus ${ }^{6}$. The descending tract continues caudally, occupying a lateral position in the dorsal funiculus of the spinal cord (Figs. 1F, 2D) where it terminates in the first cervical spinal levels.

Degenerating trigeminal fibers were not traced to the cerebellum or to any nuclei of the octavolateralis area (Fig. 1); previous claims of such projections ${ }^{6}$ cannot be supported. However, at least one octavolateralis nucleus (intermediate octavomotor nucleus, Fig. 1C) may receive trigeminal input. Dendrites of the large octavomotor cells pass through the descending trigeminal tract, and trigeminal synapses could occur on a portion of these dendrites. Golgi studies of the other ovtavolateral nuclei ${ }^{4,6}$ do not reveal dendritic invasion of the descending trigeminal tract.

The lateral line nerves in other anamniotic vertebrates terminate in the dorsal and medial nuclei of the octavolateralis area ${ }^{1,7,8}$. In lampreys, degenerating trigeminal fibers were not traced to these nuclei, thus there is no evidence that the trigeminal nerve contains a lateralis component. It seems likely that the profundus branch, believed to innervate head neuromasts, contains a component of the lateral line nerve which innervates these neuromasts as in other vertebrates ${ }^{9}$.

The descending trigeminal tract does project to the funicular nucleus as Johnston $^{6}$ claimed. However, Johnston believed that the tract ran ventral to the funicular nucleus and that trigeminal fibers turned dorsally to terminate in this nucleus. These interpretations now appear to be incorrect, as Johnston underestimated the size and extent of the descending trigeminal tract. In fact his funicular nucleus is not a separate structure from the descending trigeminal tract, but rather the nucleus and tract of the trigeminal nerve at obex levels.

Another population of cells at the obex level may, however, possess both trigeminal and spinal inputs. Neurons in the dorsal gray (dg) (Fig. IE) have apical dendrites that extend into the descending trigeminal tract as well as more ventrally into the lateral funiculus ${ }^{4,6}$. Such cells may constitute a simple dorsal column system.

A distinct descending trigeminal tract and nucleus can be recognized in lampreys, but there is scant evidence of the principal sensory and mesencephalic nuclei that exist in most vertebrates. A simple principal sensory nucleus may exist at isthmal levels, and two cellular areas bear further experimental study. There is a distinct plate of cells, medial to the entry of the trigeminal sensory root, that I have termed the isthmal alar gray (ag) (Figs. 1A, 2A). This plate is usually divided into a medial periventricular layer of the cerebellum and a lateral cerebellar nucleus. However, the lateral cells of this plate possess laterally oriented dendrites that ramify among the entering trigeminal sensory fibers ${ }^{4}$. More caudally (Figs. 1B, C, 2B) a distinct cellular crescent caps the trigeminal fibers; dorsally it comes into close contact with the cells of the trigeminal fibers ${ }^{4}$ and could represent a principal sensory nucleus or only a specialized part of the descending nucleus.

A distinct large-celled mesencepahlic nucleus of the optic tectum does not 
appear to exist in lampreys. However, one population of cells warrants further study. The medullar alar plate of lampreys is characterized by a series of large neurons scattered along its rostrocaudal extent ${ }^{10}$ (Fig. 1D). These cells are remarkably similar to the dorsal cells of the spinal cord, which are known to be primary sensory neurons ${ }^{13}$. The dorsal cells of the alar plate may constitute a rostral continuation of the spinal dorsal cells and a homologue of the 'mesencephalic' trigeminal nucleus of other vertebrates. No degenerating fibers were traced to these medullar cells in my preparations, but this does not preclude the possibility that some of these cells are associated with the trigeminal nerve. Transection of a distal neurite commonly fails to result in degenerative changes in the cells, and it is unlikely that one or two degenerating fibers could be traced across a single section in silver preparations. Other experimental methods are more likely to resolve this question.

At present there is little indication that the dorsolateral region of the lamprey hindbrain represents a 'primitive' somatic sensory column characterized by convergence of trigeminal, lateralis and octavius inputs to cells of any single nucleus. Additional experimental studies are needed, but it is likely that the lamprey medullar condition is far more similar to that of other vertebrates than was previously believed.

This research was supported in part by NIH Grant 1 R01 EY02485.

1 Boord, R. L. and Campbell, C. B. G., Structural and functional organization of the lateral line system of sharks, Amer. Zool., 17 (1977) 431-443.

2 Goodrich, E.S., Studies on the Structure and Development of Vertebrates, Macmillan, London, 1930. (Reprinted by Dover Publications, New York, 1958.)

3 Healey, E. G., The central nervous system. In M. W. Hardisty and I. C. Potter (Eds.), The Biology of Lampreys, Vol. 2, Academic Press, New York, 1972.

4 Heier, P., Fundamental principles in the structure of the brain; a study of the brain of Petromyzon fuviatilis, Acta anat. (Basel), 5, Suppl. 8 (1948) 1-213.

5 Holmgren, N., General morphology of the lateral sensory line system of the head in fish, Kvart. Svenska Vetensk. Akad. Handl., 20 (1946) 1-46.

6 Johnston, J. B., The brain of Petromyzon, J. comp. Neurol., 12 (1902) 1-106.

7 Maler, L., The acousticolateral area of bony fishes and its cerebellar relations, Brain Behav. Evol., 10 (1974) 130-145.

8 McCormick, C. A., Some connections of the ocatavolateralis area in the bowfin, Amia calva, Neurosci. Abstr., 3 (1977) 91.

9 McCready, P. J. and Boord, R. L., The topography of the superficial roots and ganglia of the anterior lateral line nerve of the smooth dogfish, Mustelus canis, J. Morph., 150 (1976) 527-538.

10 Nieuwenhuys, R., Topological analysis of the brain stem of the lamprey Lampetra fluviatilis, $J$. comp. Neurol., 145 (1972) 165-178.

11 Nieuwenhuys, R., The brain of the lamprey in a comparative perspective, Ann. N.Y. Acad. Sci., 299 (1977) 97-145.

12 Pietschmann, V., Cyclostoma Ichthya, Handbook Zoology, Vol. 6, 1933, pp. 127-547.

13 Rovainen, C. M., Physiological and anatomical studies on large neurons of the central nervous system of the sea lamprey (Petromyzon marinus). II. Dorsal cells and giant interneurons, J. Neurophysiol., 30 (1967) 1024-1042.

14 Wiitanen, J. T., Selective impregnation of degenerating axons and axon terminals in the CNS of the monkey (Macaca mulatta), Brain Research, 14 (1969) 546-548. 